\title{
Integration of virtual physician visits into a provincial 8-1-1 health information telephone service during the COVID-19 pandemic: a descriptive study of HealthLink BC Emergency iDoctor-in-assistance (HEiDi)
}

\author{
Kendall Ho MD, Helen Novak Lauscher PhD, Kurtis Stewart MSc, Riyad B. Abu-Laban MD, \\ Frank Scheuermeyer MD, Eric Grafstein MD, Jim Christenson MD, Sandra Sundhu BA
}

\section{Abstract}

Background: British Columbia, like many jurisdictions, has a health information telephone service (8-1-1) to provide callers with information by registered nurses and help them decide whether to attend an emergency department or primary care clinic, or manage their concern at home. We describe a new service, HealthLink BC Emergency iDoctor-in-assistance (HEiDi), that partnered physicians available by videoconferencing with 8-1-1 registered nurses to support callers.

Methods: From Apr. 6 to Aug. 2, 2020, all callers to the 8-1-1 telephone service (available to anyone in BC) categorized as "seek care within 24 hours" by registered nurses were eligible for referral to HEiDi. HEiDi physicians ("virtual physicians") connected directly with callers via desktop videoconferencing software, assessed their health complaint, provided advice and suggested care disposition. We conducted a descriptive study and collected demographic characteristics, health concern and disposition determined by the virtual physician.

Results: HEiDi virtual physicians provided 7687 consultations. Most patients ( $n=4439,57.8 \%$ ) were in the 20-64 age range, and $4814(62.9 \%)$ were female. Common health concerns were related to gastroenterology $(n=1275,16.6 \%)$, respiratory $(n=877$, $11.4 \%)$ and dermatology $(n=874,11.4 \%)$. From the 7531 calls with available data, $2548(33.8 \%)$ callers were advised to attempt home treatment, $2885(38.3 \%)$ to contact a primary care physician within 1 week, $1131(15.0 \%)$ to attend an emergency department immediately and $538(7.1 \%)$ to attend their primary provider now.

Interpretation: We found that virtual physicians were able to advise nearly 3 out of $4(72.1 \%)$ patients away from in-person emergency or clinic assessment and 1 in 7 (15.0\%) to seek immediate emergency department care. Virtual physicians can provide an effective complement to a provincial health telephone system.

1 he COVID-19 pandemic exposed challenges throughout Canada for residents to access health professionals and health system resources. Unfamiliarity with COVID-19 and its severity prompted many to seek educational information on symptoms to determine whether or not an urgent need existed to access acute care facilities, such as emergency departments or urgent and primary care centres. Visits to emergency departments during the pandemic decreased, ${ }^{1}$ most likely owing to fear of infection and concern over appropriateness of emergency department visits, underscoring a need for patients to have alternative ways to seek care and access health information. In addition, the rules for testing and self-isolation were complex and often changing.

In 2008, British Columbia established a province-wide telephone service (8-1-1) as an alternative to in-person assessment. Other jurisdictions (e.g., Alberta ${ }^{2}$ and the United Kingdom ${ }^{3}$ ) have similar services in which registered nurses provide advice to callers regarding their health concerns. The registered nurses can advise callers to call 9-1-1, go to an emergency department immediately, seek medical care within 24 hours, schedule an in-person appointment with their usual care provider or continue with home-based management.

Competing interests: Kendall Ho, Riyad Abu-Laban, Frank Scheuermeyer and Jim Christenson have received personal fees from HealthLink BC for work done as HEiDi virtual physicians during the course of the study. Kendall Ho has also received grants from HealthLink BC and the BC Joint Standing Committee on Rural Issues, Rural Coordination Centre of BC. Sandra Sundhu is the executive director of HealthLink BC. No other competing interests were declared.

This article has been peer reviewed.

Correspondence to: Kendall Ho, kendall.ho@ubc.ca

CMAJ Open 2021. DOI:10.9778/cmajo.20200265 
In 2016, our team conducted a pilot project and found that incorporating emergency physicians to further assess and advise callers directed to the emergency department led to a $15 \%$ decrease in emergency department visits compared with registered nurse advice alone during the same period 1 year previously. ${ }^{4}$

In March 2020, the COVID-19 pandemic led to an immediate sevenfold increase in calls to BC's 8-1-1 service, overwhelming personnel and telephone lines (S.S., unpublished data, 2020). In response and in part based on our pilot project experience, 8-1-1 rapidly initiated a "virtual physician" service - HealthLink BC Emergency iDoctor-in-assistance (HEiDi) - to assist 8-1-1 registered nurses in providing support to callers and triaging. Our aim is to describe the development and 4-month results of the HEiDi service.

\section{Methods}

\section{Design and setting}

This is a descriptive study of HEiDi, which commenced on Apr. 6, 2020, to manage calls related to COVID-19 and expanded to include all calls starting Apr. 22. The study end date was Aug. 2, 2020. We used routinely collected, anonymized data from 8-1-1. British Columbia is a province with more than 5 million residents across 5 regional health authorities. ${ }^{5}$ The 8-1-1 telephone service, managed by HealthLink $\mathrm{BC}$, which is part of the BC Ministry of Health, is available to any $\mathrm{BC}$ resident and manages about 450000 calls annually (S.S., unpublished data, 2020).

\section{Participants}

Any person in BC is able to call 8-1-1 to seek free-of-charge health information. To be referred to the HEiDi service by one of 8-1-1's registered nurses, callers must meet the following criteria: be a resident of $\mathrm{BC}$, disclose their Personal Health Number to 8-1-1 personnel (i.e., callers cannot be anonymous), have sufficient English-language proficiency (as determined by the registered nurse) or have a family member or caregiver present to translate, call during the service hours of 10:00-22:00, and be triaged as "yellow" by the registered nurse (see "The HEiDi service").

\section{The HEiDi service}

After callers are connected with a trained registered nurse, they can discuss their personal health issues, those of a family member or other individual under their care. The nurses determine callers' dispositions using the Healthwise algorithm (Healthwise Inc.) ${ }^{6}$ and their clinical judgment, triaging callers to 1 of the following HealthLink BC-determined categories: "red" to seek emergency department care immediately, "yellow" to seek care within 24 hours, "black" to seek primary care within 7 days or "green" to try home treatment.

In mid-March 2020, HealthLink BC established the HEiDi service in partnership with the BC Emergency Medicine Network, ${ }^{7,8}$ Rural Coordination Centre of $\mathrm{BC}^{9}$ and University of British Columbia Department of Emergency Medicine. HEiDi recruited 40 family and emergency physicians to work as virtual physicians, using personal contact lists and snowball recruiting; potential virtual physicians were contacted by telephone or email. Selection criteria for virtual physicians included the following: having expertise in differentiating medical conditions that could be managed in the community from those that required emergency care, and practising in different geographical locations across the province (i.e., outside of Vancouver, Victoria and the BC Lower Mainland). A virtual community of practice including all virtual physicians led by the medical director convened regularly for quality assurance purposes.

Virtual medical office assistants, situated on-site at 8-1-1 offices, received referrals from 8-1-1 registered nurses and transferred callers to connect with virtual physicians, who were located off-site. Virtual physicians initially provided 12 hours of service per day from 10:00 to 22:00, 7 days per week, and rapidly increased (on May 4) to 36 hours of combined daily service (with $>1$ physician) within 4 weeks.

As the virtual physician coverage could not accommodate all callers who connected with registered nurses, HealthLink $\mathrm{BC}$ and virtual physician leadership jointly decided on a set of caller eligibility criteria for referral from 8-1-1 registered nurses to HEiDi (see "Participants"). Callers triaged as "yellow" (seek care within 24 hours) by registered nurses were selected, as it was thought that this group of callers could potentially be given sufficient advice that may render emergency department or unscheduled same-day walk-in clinic visits within 24 hours unnecessary. "Red" callers (seek emergency department care immediately) were not included, as HEiDi was a new service, and its initiation should not delay callers accessing care.

With callers' explicit consent, virtual medical office assistants securely sent callers' synopses of their chief complaints and related health information captured by 8-1-1 registered nurses to virtual physicians via an electronic medical record system (MOIS, Bright Health). Virtual physicians initiated the consults with callers via Zoom phone calls (Zoom Video Communications, Inc.); if the clinical situation was deemed by either the caller or the virtual physician to warrant visual assessment, and with mutual agreement, a Zoom video call was initiated. Virtual physicians provided health advice to callers directly and retained or modified the disposition decision by the registered nurse based on the same classification described above. To ensure safety, virtual physicians could trigger follow-up calls by another virtual physician the next day or later to check callers' health status, ensure improvement or exclude deterioration. In addition, for quality assurance purposes, all new virtual physicians during their first 3 clinical shifts had all callers contacted by the follow-up virtual physician the next day.

\section{Data sources}

Three different data sources were used: 8-1-1's telephony system, which recorded the volume of calls received each day by service level; 8-1-1's caller encounter electronic health record, a database with a detailed record of each caller, including demographic characteristics, health problem category, 
nurse triage decision, and, specific to HEiDi, the virtual physician triage decision and clinical notes; and, from May 18 onward, MOIS data extracts, which contained information related to virtual physician consultation characteristics (e.g., caller waiting time and duration of call). Data were extracted from all 3 sources by a member of HealthLink BC's Business Intelligence Unit (who was not part of the study team) following their standard, internal processes. Data extracts were checked for accuracy by this same person from HealthLink $\mathrm{BC}$, then deidentified and shared with the study team.

\section{Statistical analysis}

We focused on both program and caller outcomes relevant to HEiDi, including the following: call volumes, particularly the busiest days; characteristics of callers accessing HEiDi (i.e., age group, sex, health concern, whether the concern is related to COVID-19, health authority and having a usual care provider); characteristics of the virtual consultations (i.e., consultation duration, wait times and number of consultations done by virtual physicians); and the proportion of callers diverted from seeking care within 24 hours (as defined by the percentage of callers triaged "yellow" by virtual physicians compared with registered nurses). We additionally standardized calls by each geographic health authority's population to compare the number of calls per 100000 residents (i.e., dividing the number of calls by the population, then multiplying by 100000$).{ }^{10}$ The number of virtual physician follow-up calls and data collected during these calls were excluded from analyses, except for whether the caller had a usual care provider, as this information was only recorded during follow-up calls for that subset of callers.

We used R (version 4.0.2, R Core Team) for the analysis, with results expressed descriptively as means and standard deviations (SDs) if normally distributed, or medians and interquartile ranges (IQRs) if nonnormally distributed. We used $\chi^{2}$ tests to compare distributions between categorical variables and $t$ tests to compare normally distributed variables (no comparisons of nonnormally distributed variables were done).

\section{Ethics approval}

As this descriptive study was for quality assurance and improvement purposes and used routinely collected anonymized data, an ethics waiver was provided by the University of British Columbia's research ethics board, in keeping with the Tri-Council Policy Statement: Ethical Conduct for Research Involving Humans. ${ }^{11}$

\section{Results}

Table 1 summarizes the demographic characteristics of patients $(n=7687)$. Callers were more likely to be female, and $4439(57.8 \%)$ were adults aged 20-64 years. Health concerns encompassed a range of 23 standard problem categories used by 8-1-1 registered nurses (Appendix 1, Supplementary Table 1, available at www.cmajopen.ca/content/9/2/E635/ suppl/DC1); 3839 (49.9\%) were 1 of 4 categories and, based

\begin{tabular}{|c|c|}
\hline Characteristic & No. $(\%)^{*}$ \\
\hline Sex, female & $4814(62.9)$ \\
\hline Missing or unknown & 37 \\
\hline \multicolumn{2}{|l|}{ Age group, yr } \\
\hline$<1$ & $544(7.1)$ \\
\hline $1-4$ & $721(9.4)$ \\
\hline $5-19$ & $887(11.5)$ \\
\hline 20-39 & $2603(33.9)$ \\
\hline $40-64$ & $1836(23.9)$ \\
\hline$>64$ & $1090(14.2)$ \\
\hline Missing or unknown & 2 \\
\hline \multicolumn{2}{|l|}{ Health authority } \\
\hline Fraser & 2688 (35.9) \\
\hline Interior & $1172(15.6)$ \\
\hline Northern & $435(5.8)$ \\
\hline Vancouver Coastal & $1648(22.0)$ \\
\hline Vancouver Island & $1549(20.7)$ \\
\hline Missing or unknown & 195 \\
\hline \multicolumn{2}{|l|}{ Health concern category } \\
\hline Gastroenterology (digestive) & $1275(16.6)$ \\
\hline Respiratory & $877(11.4)$ \\
\hline Dermatology (skin, hair, nails) & $874(11.4)$ \\
\hline $\begin{array}{l}\text { Musculoskeletal (bone, muscle, } \\
\text { joint) }\end{array}$ & $813(10.6)$ \\
\hline $\begin{array}{l}\text { Caller's health concern related to } \\
\text { COVID-19 }\end{array}$ & 1169 (39.3)† \\
\hline Missing or unknown & 4710 \\
\hline Caller has a usual care provider & 1228 (81.3)‡ \\
\hline Missing & 133 \\
\hline No follow-up & 6043 \\
\hline \multicolumn{2}{|c|}{$\begin{array}{l}\text { Note: } \mathrm{HEiDi}=\text { HealthLink BC Emergency iDoctor-in-assistance. } \\
\text { "Percentages were calculated after removing missing, unknown or not applicable } \\
\text { data from the denominator. } \\
\text { †Only a subset of } 2977 \text { HEiDi calls had available data on whether they were } \\
\text { related to COVID-19. } \\
\text { fOnly callers receiving a subsequent follow-up call from a virtual physician are } \\
\text { asked if they have a usual care provider; } 1511 \text { callers received a follow-up call } \\
\text { and answered this question. }\end{array}$} \\
\hline
\end{tabular}

on a subset of 2977 calls with available data, 1169 (39.3\%) were related to COVID-19. The distribution of age groups differed significantly by sex $(p<0.001)$, with female patients more likely to be age $20-39$ years $(n=1841,38.2 \%$ v. $n=743$, $26.2 \%)$ and males more likely to be age $0-4$ years $(n=663$, $23.4 \%$ v. $n=600,12.5 \%$ ) (Appendix 2, Supplementary Table 2, available at www.cmajopen.ca/content/9/2/E635/ suppl/DC1).

Although 2688 (35.9\%) absolute calls came from 1 regional health authority (Fraser), calls standardized by population showed that the number of calls per 100000 residents was 180.37 for Vancouver Island, 152.99 for Northern, 


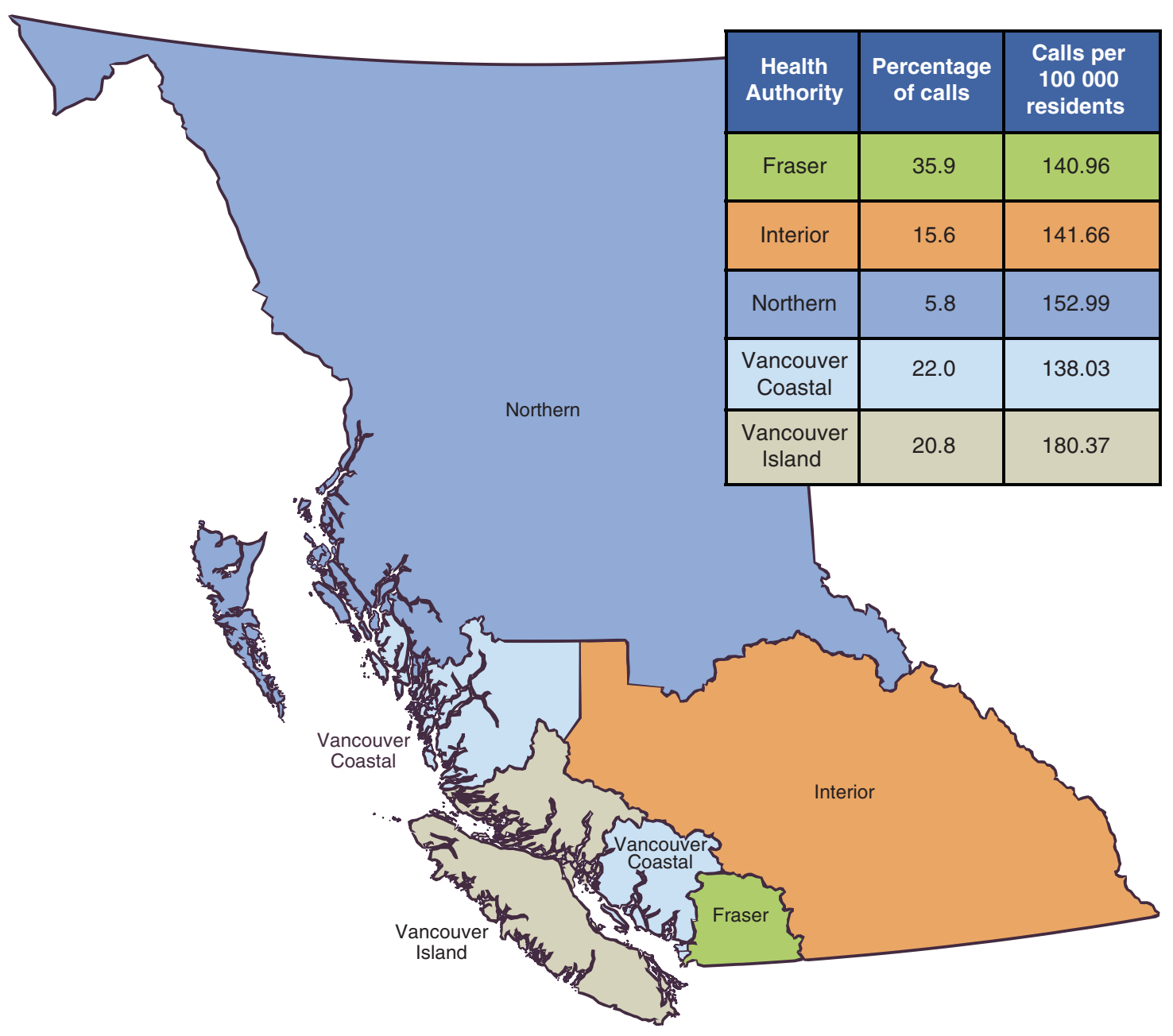

Figure 1: Summary of percentage of calls and population-standardized number of calls by BC Health Authority. This map is produced from the "Health Authority Boundaries" data published by the Ministry of Health - Health Sector Information Analysis and Reporting. Licensed under Open Government Licence — British Columbia (https://catalogue.data.gov.bc.ca/dataset/health-authority-boundaries).

141.66 for Interior, 140.96 for Fraser and 138.03 for Vancouver Coastal (Figure 1). The distribution of age groups differed significantly by health authority $(p<0.001)$, but sex was similar across health authorities $(p=0.4)$ (Appendices 3 and 4, Supplementary Tables 3 and 4, available at www.cmajopen.ca/ content/9/2/E635/suppl/DC1).

Based on 5659 consultations with available data in MOIS since its implementation on May 18 to Aug. 2, 2020, 5538 unique callers accessed HEiDi, with 112 patients having multiple consultations (excluding follow-ups) within the reporting period. Of those, 107 (95.5\%) had 2 consultations and 5 $(4.5 \%)$ had 3 or more.

\section{Call volumes}

During the 17-week study period, 8-1-1 registered nurses triaged 21441 patients as "yellow," of whom 7845 (36.6\%) were referred to HEiDi and 7687 (98.0\%) of those received a virtual consultation. Figure 2 shows the flow of callers through 8-1-1 and HEiDi. Figure 3 shows the daily and cumulative call volumes of HEiDi for the reporting period. The mean daily number of calls was 74.84 (SD 12.62), and the range was 12 (Apr. 6) to 106 (July 12). On average, Saturday and Sunday were the busiest days of the week, and Monday and Tuesday were the slowest (Appendix 5, Supplementary Table 5, available at www.cmajopen.ca/content/9/2/E635/suppl/DC1); weekends (mean 73.32, SD 21.91, calls per day) were busier than weekdays (mean 62.42, SD 20.21, calls per day; $p=0.02$ ).

\section{Virtual physician consultation characteristics}

After speaking with an 8-1-1 registered nurse, most callers $(n=6666,85.0 \%)$ waited 1 minute or less before their call was answered by a HEiDi virtual medical office assistant (median 0.05, IQR 0.03-0.32, min). After speaking with the virtual medical office assistant, callers waited a median time 


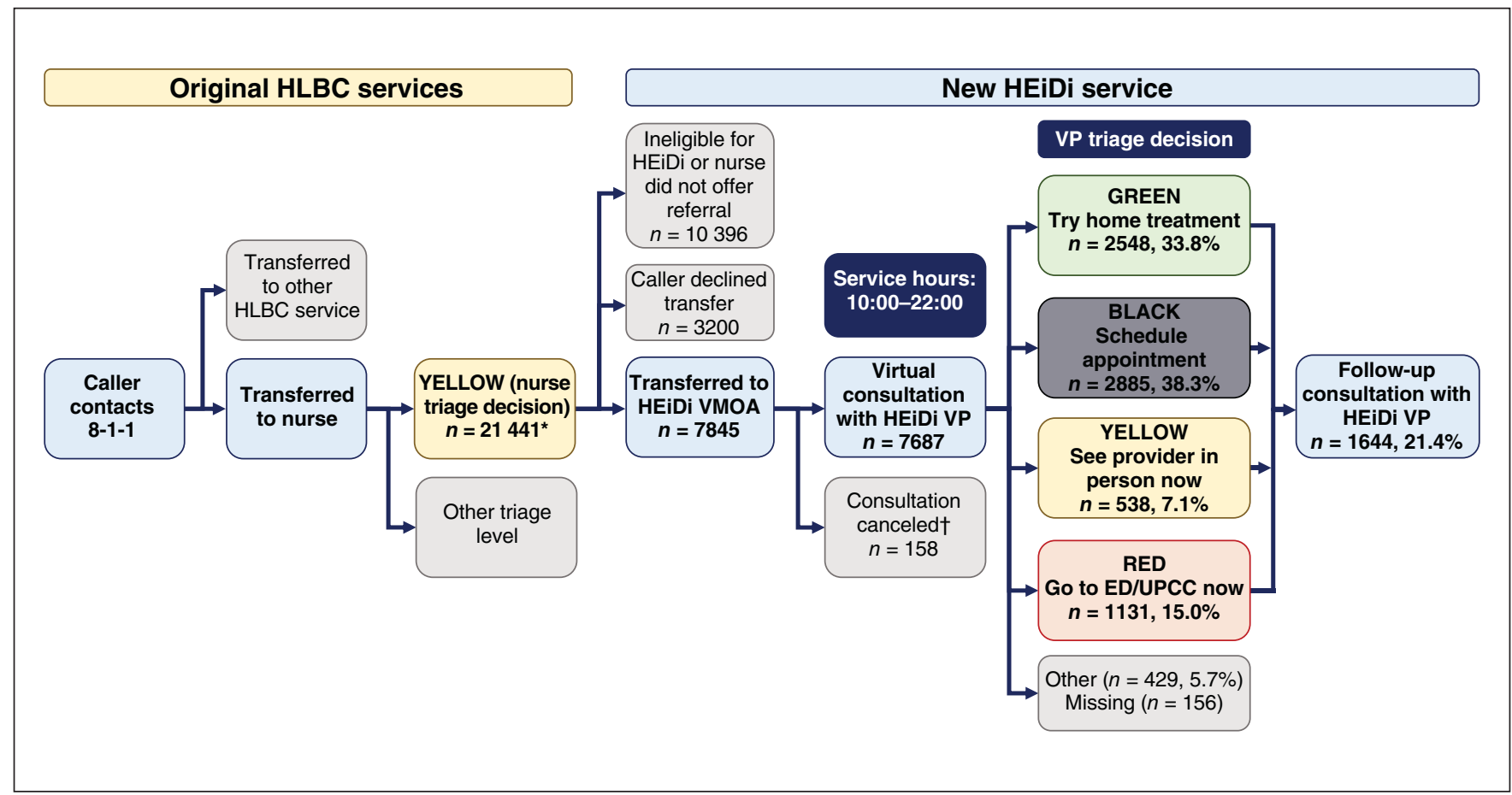

Figure 2: Flow of callers through 8-1-1 and HealthLink BC Emergency iDoctor-in-assistance (HEiDi) service. Note: ED = emergency department, $\mathrm{HLBC}=$ HealthLink BC, UPCC $=$ urgent and primary care centre, VMOA = virtual medical office assistant, VP = virtual physician. ${ }^{*}$ Nurse call numbers reported here are only for the 12-hour period of the HEiDi service (Apr. 6 to Aug. 2, 2020, 10:00 to 22:00). †Consultation could be cancelled by the patient or VMOA for several reasons (e.g., VMOA disconnected, patient disconnected, patient does not require service, incorrect record, no associated record).

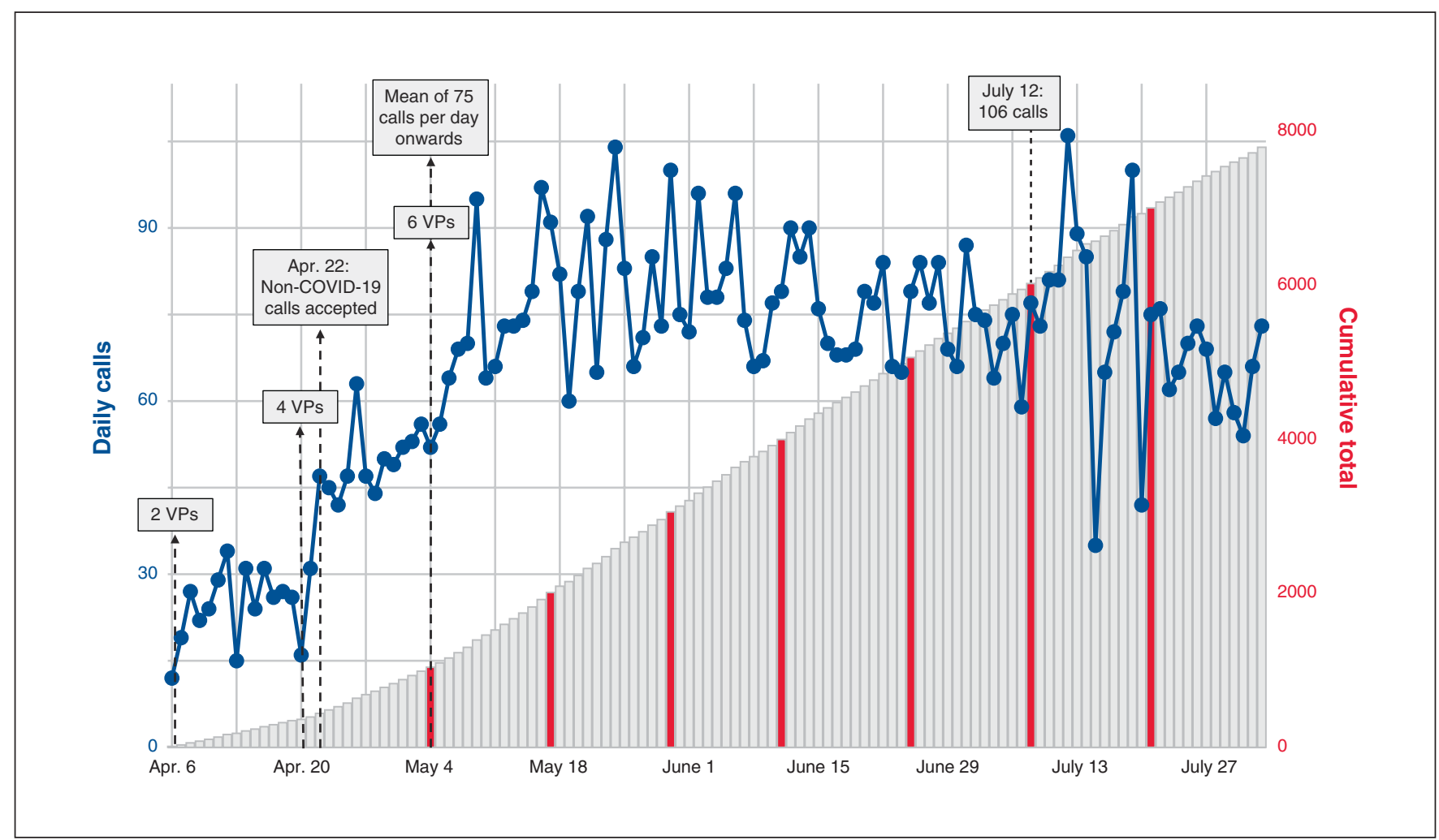

Figure 3: Daily (blue) and cumulative (grey) call volumes to HealthLink BC Emergency iDoctor-in-assistance (HEiDi). Red bars indicate when cumulative calls reached a multiple of 1000 (i.e., 1000 to 7000 ) calls. Note: VP = virtual physician. 
of 28 (IQR 19-44) minutes before the virtual physician contacted them to begin the consultation. Consultations with virtual physicians lasted a median time of 18 (IQR 13-24) minutes. Based on 5659 consultations with available MOIS data, 996 (17.6\%) consultations used Zoom video. Virtual physicians completed a mean of 12.29 (SD 3.79) consultations per 6-hour shift.

\section{Virtual physician triage decision}

Of the 7687 patients assessed by a virtual physician, 7531 (98.0\%) had available data on the virtual physician's triage decision. Of those, 2548 (33.8\%) were advised to attempt home treatment and 2885 (38.3\%) were advised to contact a primary care physician within 1 week, resulting in a "downgrade" of triage level for 5433 (72.1\%) callers (Figure 2). A further $1131(15.0 \%)$ were advised to attend an emergency department or urgent and primary care centre immediately, and $538(7.1 \%)$ were advised to attend their primary care provider now. The distribution of virtual physician triage decisions significantly differed by health authority $(p=0.004)$, with Northern having the lowest "green" triage proportion and Interior having the highest "red" triage proportion (Appendix 6, Supplementary Table 6, available at www. cmajopen.ca/content/9/2/E635/suppl/DC1, and Appendix 7, Supplementary Figure 1, available at www.cmajopen.ca/ content/9/2/E635/suppl/DC1).

\section{Interpretation}

Our early descriptive study of HEiDi during its first 17 weeks of service showed that virtual physicians can handle a high volume of calls (7687 consultations provided) and down-triage $72 \%$ of patients to a less urgent care disposition. Studies involving American gastroenterology and French otolaryngology patients during the COVID-19 pandemic reported that callers favourably rated telemedicine options replacing inperson appointments. ${ }^{12,13}$

The results of this descriptive study indicate that, by partnering virtual physicians with 8-1-1 registered nurses, a substantial percentage of patients and callers can be advised away from urgent in-person visits to emergency departments or unscheduled same-day visits to walk-in clinics, compared to advice by registered nurses alone. This on-demand phone and videoconferencing service was easily accessible and convenient for all BC residents, available 7 days per week anywhere in the province, including remote communities.

During the first wave of the COVID-19 pandemic, HEiDi physician advice had substantial potential to help divert patients away from urgent emergency department visits, thereby preserving emergency departments and urgent and primary care centre capacity to treat those who needed acute care services, decreasing congestion and overcrowding of emergency departments or walk-in clinics, and improving appropriate use of acute care services. Moreover, a virtual approach protected individuals from unnecessary potential exposure to SARSCoV-2 and other infections in emergency department or urgent and primary care centre waiting rooms.
Although our early evaluation does not scrutinize circumstances in selecting video versus telephone consults for caller encounters, this will be important to evaluate in future research to examine the comparative benefits of telehealth modality. Further, our results must be interpreted with caution, as our design cannot demonstrate a causal link between virtual physician intervention and reduction in emergency department volumes. Nevertheless, the downgrading of triage after virtual physician intervention offers the potential of a positive signal and warrants future research into HEiDi's impact on appropriate emergency department utilization.

Appropriate triaging of 8-1-1 callers also encouraged those patients who required acute care to present to an emergency department immediately, while reassuring patients with conditions that could be managed at home or by their community-based health care services. In this respect, the HEiDi service was designed to preserve the primary care system to serve callers' needs longitudinally by health professionals familiar to them.

Although this first implementation of HEiDi addressed only the "yellow" (seek care within 24 hours) disposition, ongoing evaluation will provide important information to guide the intended incorporation of other call categories as appropriate, in particular the higher risk "red" callers (seek emergency department care immediately). From the health system's perspective, this preliminary descriptive study suggests that HEiDi is a consistent, reliable and provincially accessible approach for timely access of health advice to all callers. The virtual physician workforce can contract or expand based on caller volumes, providing staffing flexibility for pandemic-related volume changes. HEiDi supported the appropriate triaging of callers through interprofessional collaboration with 8-1-1 registered nurses and virtual medical office assistants, such that only calls requiring physician intervention were answered by HEiDi. Conceivably, this could result in cost-effectiveness by matching physician coverage with anticipated caller volumes.

\section{Limitations}

This is a preliminary report of a physician-assisted, nursemanaged telephone service in a single province during a pandemic; thus, results may differ in other settings or times. Callers outside of BC or those who did not speak English were not served by this program, and results may differ in such groups. We are currently developing a study to collect linked administrative data and assess actual emergency department, hospital or family doctor visits and deaths of HEiDi callers. This will allow us to more accurately track caller adherence to virtual physician triage and callers' ultimate outcomes.

During this COVID-19 period, it was not uncommon for primary care physician services to close, so this could increase HEiDi's rates of referral to emergency departments and urgent and primary care centres compared with periods when more comprehensive primary care was available. Some patients may benefit from or prefer video interactions, but our data currently do not allow us to characterize this group. At 
this stage, we cannot estimate the direct and indirect costs and benefits of the program beyond the outlined results.

\section{Conclusion}

Our early descriptive study of HEiDi during its first 17 weeks of service showed that virtual physicians can add substantial and flexible capacity to a provincial 8-1-1 service. The addition of virtual physicians to the service resulted in a substantial proportion of callers advised away from seeking immediate in-person medical attention and advised to accelerate urgent care when appropriate. Our innovative use of information technology enabling virtual physician consultations supported residents' equitable access to needed health care advice and information, and merits consideration in other similar public telephone access systems, during and beyond pandemics. In future research, we intend to link our 8-1-1 data with emergency department and practitioner data to assess patient safety, system utilization and cost-effectiveness.

\section{References}

1. Canadian emergency department visits drop 25\% in early weeks of COVID-19 pandemic. Ottawa: Canadian Institute for Health Information; 2020. Available: www.cihi.ca/en/canadian-emergency-department-visits-drop-25-in-early-weeks -of-covid-19-pandemic (accessed 2020 Sept. 1).

2. Health Link 811. Edmonton: Alberta Health Services; 2020. Available: www. albertahealthservices.ca/findhealth/service.aspx? Id=1001957\&facilityId=1011654 (accessed 2020 Sept. 1).

3. When to use 111. London (UK): National Health Service. Available: www.nhs. uk/using-the-nhs/nhs-services/urgent-and-emergency-care/nhs-111 (accessed 2020 Sept. 1).

4. Grafstein E, Abu-Laban RB, Wong B, et al. LO16: Safety and efficiency of emergency physician supplementation in a provincially nurse-staffed telephone service for urgent caller advice. CFEM 2017;19(Suppl 1):S32-3.

5. Quarterly population highlights. British Columbia Ministry of Health, Office of the Provincial Health Officer; 2021. Available: www2.gov.bc.ca/assets/gov/ data/statistics/people-population-community/population/population_highlights 2020q4.pdf (accessed 2021 Apr. 13).

6. Healthwise helps you make better health decisions. Boise (ID): Healthwise: 2021. Available: www.healthwise.org/specialpages/legal/abouthw/en (accessed 2021 Jan. 25).

7. Abu-Laban RB, Drebit S, Lindstrom RR, et al. The British Columbia Emergency Medicine Network: a paradigm shift in a provincial system of emergency care. Cureus 2018;10:e2022.

8. Marsden J, Drebit S, Lindstrom RR, et al. The BC Emergency Medicine Network: evaluation approach and early findings. BCMF 2019;61:164-71.

9. Rural Coordination Centre of BC (RCCBC) [main page]. Vancouver: RCCBC. Available: https://rccbc.ca (accessed 2020 Sept. 1).

10. British Columbia: population estimates. How to use the population estimates application. BC Stats. Available: https://bcstats.shinyapps.io/popApp (accessed 2020 Sept. 1).

11. Tri-Council Policy Statement: ethical conduct for research involving bumans - TCPS 2 (2018). Ottawa: Secretariat on Responsible Conduct of Research, Canadian
Institutes of Health Research; modified 2020 Feb. 19. Available: https://ethics. gc.ca/eng/policy-politique_tcps2-eptc2_2018.html (accessed 2020 Sept. 1)

12. Fieux M, Duret S, Bawazeer N, et al. Telemedicine for ENT: effect on quality of care during COVID-19 pandemic. Eur Ann Otorbinolaryngol Head Neck Dis 2020;137:257-61.

13. Dobrusin A, Hawa F, Gladshteyn M, et al. Gastroenterologists and patients report high satisfaction rates with telehealth services during the novel coronavirus 2019 pandemic. Clin Gastroenterol Hepatol 2020;18: 2393-7.e2.

Affiliations: Digital Emergency Medicine (Ho, Novak Lauscher, Stewart), Department of Emergency Medicine, and Department of Emergency Medicine (Ho, Novak Lauscher, Abu-Laban, Grafstein, Christenson), Faculty of Medicine, The University of British Columbia; BC Emergency Medicine Network (Ho, Abu-Laban, Christenson); Department of Emergency Medicine (Scheuermeyer), St Paul's Hospital and The University of British Columbia; Centre for Health Evaluation and Outcome Sciences (Scheuermeyer), Vancouver, BC; HealthLink BC (Sundhu), Primary Care Division, Ministry of Health, Victoria, BC

Contributors: Kendall Ho, Eric Grafstein and Sandra Sundhu contributed substantially to the conception and design of HEiDi, and Helen Novak Lauscher, Kurtis Stewart, Riyad Abu-Laban, Frank Scheuermeyer and Jim Christenson contributed substantially to refinement of the design. Kendall Ho, Helen Novak Lauscher, Kurtis Stewart and Sandra Sundhu led the data acquisition and initial data analysis, and all authors contributed to subsequent data analysis and interpretation. Kendall Ho, Helen Novak Lauscher and Kurtis Stewart drafted the article, and all other authors participated in revising it critically for important intellectual content. All authors gave final approval of the version to be published and agreed to act as guarantors of the work.

Funding: The HEiDi service is funded by the BC Ministry of Health through HealthLink BC. This evaluation was funded by BC's Joint Standing Committee on Rural Issues. Members from both funding organizations participated in the cocreation of the evaluation framework. All data analysis and interpretation were done by the study team members. The HealthLink BC leadership participated in the drafting of the manuscript and decision to submit for publication, as 1 member is a coauthor (Sandra Sundhu).

Content licence: This is an Open Access article distributed in accordance with the terms of the Creative Commons Attribution (CC BY-NC-ND 4.0) licence, which permits use, distribution and reproduction in any medium, provided that the original publication is properly cited, the use is noncommercial (i.e., research or educational use), and no modifications or adaptations are made. See: https://creativecommons.org/licenses/ by-nc-nd/4.0/

Data sharing: Data reported here are available from the corresponding author on reasonable request to replicate our analysis. Requisite permissions must be obtained from HealthLink BC. Please contact the corresponding author to facilitate this process.

Supplemental information: For reviewer comments and the original submission of this manuscript, please see www.cmajopen.ca/content/9/2/ E635/suppl/DC1. 